\title{
A rare case of postpartum ovarian vein thrombosis
}

\section{Sujatha Narayanmoorthy*, Parvathy Ganesan, Radhamany Ramanan}

Department of Obstetrics \& Gynaecology, Amrita Institute of Medical Sciences, Ponekkara, Kochi-682041, Kerala, India

Received: 15 March 2015

Accepted: 19 April 2015

\section{*Correspondence:}

Dr. Sujatha Narayanmoorthy,

E-mail: nsujatha88@yahoo.co.in

Copyright: ( ) the author(s), publisher and licensee Medip Academy. This is an open-access article distributed under the terms of the Creative Commons Attribution Non-Commercial License, which permits unrestricted non-commercial use, distribution, and reproduction in any medium, provided the original work is properly cited.

\begin{abstract}
Ovarian vein thrombosis is an uncommon life threatening postpartum complication. This septic thrombophlebitis may have a vague clinical picture which has to be promptly thought of in any woman with spiking fever, abdomen pain and raised total count especially in the puerperal period. We report a case of a thirty one year old lady in whom this condition was recognized with clinical acumen, computed tomography and Doppler sonography. Thrombolysis was the modality of treatment thereby avoiding the potential complications.
\end{abstract}

Keywords: Postpartum, Ovarian vein thrombosis, Management, Heparin

\section{INTRODUCTION}

Ovarian Vein Thrombosis (OVT) is a rare fatal entity occurring in the puerperal period complicating $0.05 \%$ to $0.18 \%$ of pregnancies. ${ }^{1}$ It is also associated with a variety of pelvic conditions like pelvic surgeries, inflammatory disease and malignancies. This condition has preponderance to the right ovarian vein. Prompt diagnosis of postpartum OVT is important to prevent its serious complications like pulmonary embolism, sepsis and mortality. We report a case of right OVT in a thirty one year old woman following a full term normal delivery.

\section{CASE REPORT}

A thirty one year old parous lady was referred to Amrita Institute of Medical Science, India on the ninth day following a term vaginal delivery with puerperal pyrexia. She had an uneventful antenatal period. At thirty nine weeks of gestation labour was induced with misoprostol and oxytocin. Following the delivery of the foetus manual removal of placenta was done under anesthesia. She developed spikes of fever with chills on the second post natal day. There was no associated vaginal bleeding, abdominal pain, vomiting or altered bowel or bladder habits. Baseline investigations were reported to be normal. She failed to respond to empirical antibiotics which were cefixime and amoxicillin-clavulanic acid.

On day nine, a multidisciplinary approach by the departments of Obstetrics and Gynecology, General Medicine and Gastrointestinal Surgery was sought at our hospital for further evaluation and management. On examination, the patient's general condition was fair. Temperature recordings revealed recurrent spikes of fever with no definitive pattern. She was normotensive. There was no pallor or pedal edema. Per abdomen examination showed right iliac fossa tenderness radiating to the back and groin. Local episiotomy and vaginal examination were normal. Ultrasonography (USG) of abdomen, pelvis and neck reported no abnormalities. Baseline investigations showed raised counts of 12900 cells $/ \mathrm{mm}$ and C Reactive Protein (CRP) of 238 units. Urine, blood and vaginal swab culture and sensitivity grew no organisms. Other infective causes of pyrexia were excluded with Widal test, Enzyme-Linked ImmunoSorbent Assay (ELISA) of leptospira and dengue, and the test for malarial parasite. She continued to fail to respond to empirical antibiotics cefoperazone sulbactam and metronidazole given in our hospital. 
Suspecting a pelvic vein thrombosis, a Multi-Detector Computed Tomography (MDCT) of abdomen was done that showed a right OVT extending up to the Inferior Vena Cava (IVC). Figure 1 shows the MDCT of our patient with the right OVT indicated by an arrow. Ovarian Doppler study showed occlusion of the right ovarian vein drainage into the IVC. She was started on 60 mg enoxaparin subcutaneously once a day. During her course of stay, she improved significantly and after being afebrile for forty eight hours she was discharged. On her first month review visit, she was found to be asymptomatic. Ovarian Doppler study was repeated that showed the absence of thrombus. The dose of enoxaparin was adjusted.

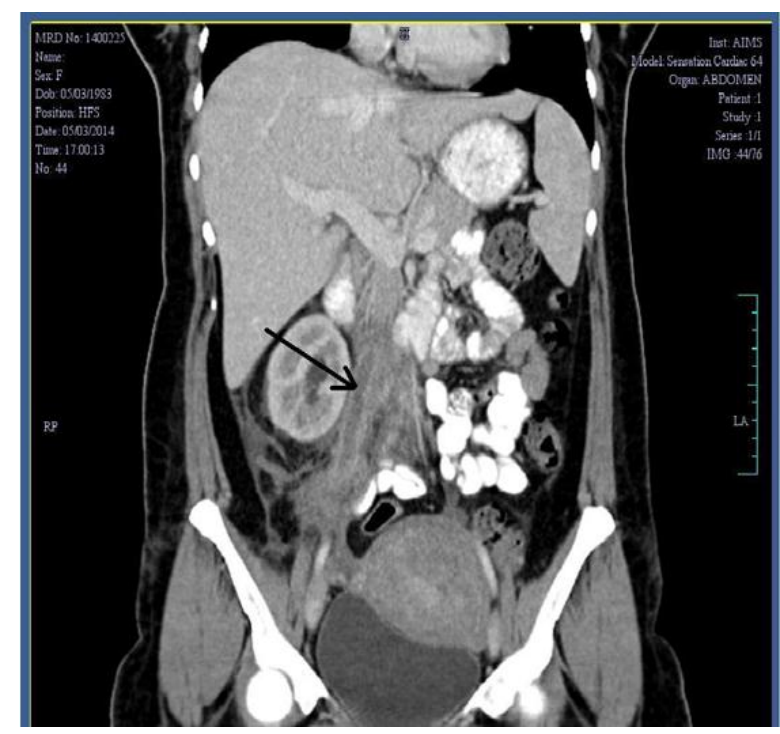

Figure 1: MDCT image with the right ovarian vein thrombosis indicated by a black arrow mark.

\section{DISCUSSION}

Postpartum OVT is a well-known yet an uncommon life threatening complication. It may be preceded by an abortion, vaginal delivery or a cesarean section. Salomon et al. ${ }^{2}$ stated that the risk of its development is maximal following a cesarean section for a twin gestation. It affects one in six hundred to one thousand two hundred pregnancies. ${ }^{1}$ Though it typically presents with fever, dull aching abdominal pain and leukocytosis, its characteristics may be vague and variable.

The right ovarian vein is noted to be affected in $80 \%$ of the cases. Various hypotheses have been postulated for the same. Calderwood et al. ${ }^{3}$ stated that the right ovarian vein gets easily compressed due to the dextrorotation of the uterus and also due to its entry into the IVC at an acute angle. Savander et al. ${ }^{4}$ concluded that the right ovarian vein is longer than the left and has many valves within its length, which increases the risk of thrombus formation. Literature ${ }^{5}$ also opines that the right ovarian vein is predisposed to thrombosis because there is an anterograde flow in the right ovarian vein, while a retrograde flow is noted in the left ovarian vein immediately after delivery.

Virchow's triad and pregnancy itself being a thrombotic state governs its pathophysiology. Differential diagnosis may include septic pelvic thrombophlebitis, pyelonephritis, urinary tract infection, adnexal torsion, intestinal volvulus, puerperal endometritis and tuboovarian abscess.

Though USG abdomen may be used to diagnose a postpartum OVT, confounding factors like overlying gas shadow limits its accuracy. In our patient USG abdomen could not pick up the thrombosis. An abdominopelvic MDCT or Magnetic Resonance Imaging (MRI) may be used for a confirmatory diagnosis. The coronal sections of MDCT provide information on the extent of the thrombus. A thick-walled enlarged ovarian vein with rim enhancement and central hypodensity are the features seen in a Computed Tomography (CT) scan that suggests OVT. An adjuvant Doppler study may be done to look for the patency of the other veins specifically IVC.

Mainstay of treatment remains to be anticoagulants and broad spectrum antibiotics. While some authors suggest warfarin as the anticoagulant of choice, others suggest low molecular weight heparin for thrombolysis. Garcia et al. ${ }^{6}$ stated that the thrombus resolves within seven to fourteen days from the commencement of the therapy. Literature does not give a definitive protocol on the type, dosage, or duration of anticoagulation therapy. While some believe in short course of anticoagulation, others have suggested continuing treatment for three to six months. ${ }^{6,7}$ Our patient took a three month course of Low Molecular Weight (LMW) heparin.

Our experience emphasizes the need for early diagnosis of postpartum OVT as it is associated with increased complication and mortality. Any puerperal fever not responding to antibiotics and is associated with abdominal pain should prompt us to image the abdomen and look for thrombosis. Anticoagulation is regarded the gold standard treatment. More studies must be done in future to give us a definite protocol on the management of this entity.

\section{ACKNOWLEDGEMENTS}

Dr. Sandya, Clinical Associate Professor, department of Radiodiagnosis, Amrita Institute of Medical Science, is thanked for the CT images provided. I would like to thank Vishal Sridhar for the technical assistance in the preparation of this manuscript.

\section{Funding: No funding sources}

Conflict of interest: None declared

Ethical approval: The study was approved by the ethics committee on human experimentation (institutional and national) and with the Helsinki Declaration of 1975, as revised in 2008 (5). 


\section{REFERENCES}

1. Basili G, Romano N, Bimbi M, Lorenzetti L, Pietrasanta D, Goletti O. Postpartum ovarian vein thrombosis. JSLS. 2011;15:268-71.

2. Salomon O, Dulitzky M, Apter S. New observations in postpartum ovarian vein thrombosis: experience of a single center. Blood Coagul Fibrunolsys. 2010;21:16-9.

3. Calderwood CJ, Jamienson R, Greer IA. Gestational related changes in the deep venous system of the lower limb on light reflection rheography in pregnancy and the puerperium. Clin Radiol. 2007;62:1174-9.
4. Savander SJ, Otero RR, Savander BL. Puerperal ovarian vein thrombosis: evaluation with CT, US, and MR imaging. Radiology. 1988;167:637-9.

5. Simons GR, Piwnica-Worms DR, Goldhaber SZ. Ovarian vein thrombosis. Am Heart J. 1993;126:6417.

6. Garcia J, Aboujaoude R, Apuzzio J, Alvarez JR. Septic pelvic thrombophlebitis: diagnosis and management. Infect Dis Obstet Gynecol. 2006;2006:15614.

7. Al-toma A, Heggelman BG, Kramer MH. Postpartum ovarian vein thrombosis: report of a case and review of the literature. Neth J Med. 2003;61:334-6.

DOI: $10.18203 / 2320-1770 . i j r \operatorname{cog} 20150117$

Cite this article as: Narayanmoorthy S, Ganesan P, Ramanan R. A rare case of postpartum ovarian vein thrombosis. Int J Reprod Contracept Obstet Gynecol 2015;4:878-80. 